\title{
Coupling between counterpropagating cladding modes in fiber Bragg gratings
}

\author{
D. Sáez-Rodriguez, J. L. Cruz,** A. Díez, and M. V. Andrés \\ Department of Applied Physics and Electromagnetism, University of Valencia, Dr. Moliner 50, Burjassot 46100, Spain \\ *Corresponding author: cruz@uv.es \\ Received December 23, 2010; revised February 21, 2011; accepted March 23, 2011; \\ posted March 24, 2011 (Doc. ID 140115); published April 15, 2011

\begin{abstract}
We present an experimental demonstration of energy transfer between counterpropagating cladding modes in a fiber Bragg grating (FBG). A strong FBG written in a standard photosensitive optical fiber is illuminated with a single cladding mode, and the power transferred between the forward propagating cladding mode and different backward propagating cladding modes is measured by using two auxiliary long period gratings. Resonances between cladding modes having $30 \mathrm{pm}$ bandwidth and $8 \mathrm{~dB}$ rejection have been observed. ( 2011 Optical Society of America

OCIS codes: $\quad 060.3735,060.2400,060.2310$.
\end{abstract}

After the first observation of photosensitivity in optical fibers [1] and the first demonstration of modal coupling in periodic structures [2], fiber Bragg gratings (FBGs) and long period gratings (LPGs) in single-mode fibers have been extensively used in most fields related to optical fiber technology [3]. Gratings consist of a periodic modulation of the refractive index of the fiber core, which is able to couple the energy between the forward and the backward core mode of a single-mode fiber (in the case of a FBG) or between copropagating modes of different order (in the case of an LPG) [늑] can couple energy to counterpropagating cladding modes, because the overlap integral between cladding and core modes, at the core region where the photosensitive material is commonly located, does not vanish. Thus, a number of tools are available to transfer energy between modes; however, the development of an efficient mode engineering requires new ways to increase the ability to accurately control the excitation of specific modes and the amount of power that is transferred. There are already some examples in which in-fiber mode engineering achieved by combining FBG and LPG has demonstrated devices with new and interesting properties [으물.

Currently, Bragg gratings are being used to excite the cladding modes, and combinations of Bragg gratings with LPGs have been developed to achieve controllable power transfer between these modes. The operation principle is the interaction between core and cladding modes in the grating as demonstrated in $[11,12]$. These devices have been proposed as reflectors for large modal area lasers $[13,14]$ and as sensors or actuators [8-10,15]. For these kinds of applications, there is a need to investigate the transfer of energy specifically between cladding modes in order to pave the way for future developments.

Besides core-to-core and core-to-cladding coupling, FBGs can potentially couple energy between counterpropagating cladding modes, and fibers with a photosensitive cladding have been proposed theoretically to improve the weak overlap between cladding modes in the fiber core [16]. Despite the fact that strong gratings previously fabricated might couple energy between cladding modes, an experimental study has not been reported so far.

In this Letter, we present what we believe is the first experimental demonstration of coupling between counterpropagating cladding modes in FBGs.
In our experiment, a strong Bragg grating is illuminated with a single cladding mode $\left(\mathrm{LP}_{0 k}\right)$ generated by an $\mathrm{LPG}$, as illustrated in Fig. 1. The Bragg grating couples energy from the forward traveling cladding mode to the backward core mode $\left(\mathrm{LP}_{01}\right)$, as well as to the backward cladding modes. A second LPG identical to the first one recouples the forward propagating cladding mode into the fiber core. Therefore, the transmission spectrum reveals both cladding-to-cladding and cladding-to-core resonances at the wavelengths coupled by the Bragg grating.

All the gratings were inscribed in a boron-codoped fiber provided by Fibercore, Ltd. (product number PS1250/1500), the fiber had a NA of 0.12 and a cut-off wavelength of $1200 \mathrm{~nm}$. Gratings were inscribed by scanning the fiber by a doubled argon laser with UV power of $100 \mathrm{~mW}$.

The LPGs were written point by point, focusing the beam trough a $50 \mu \mathrm{m}$ wide slit. Both LPGs had a period of $267 \mu \mathrm{m}$ and a length of $1 \mathrm{~cm}$. The two gratings were located $16.0 \mathrm{~cm}$ apart. Figure 2(a) shows the gratings' spectra measured by a spectrum analyzer with $1 \mathrm{~nm}$ resolution; the first grating has a resonant band at $1525.8 \mathrm{~nm}$ with $35.6 \mathrm{~nm}$ bandwidth at $10 \mathrm{~dB}$. The transmission notch has a maximum attenuation of $30 \mathrm{~dB}$. The second LPG reverts the cladding mode energy into the fiber core, and the pair of gratings has a useful band of $29 \mathrm{~nm}$ with an insertion loss of $0.7 \mathrm{~dB}$ and a ripple near $1 \mathrm{~dB}$. The resonant wavelengths of the different cladding modes were theoretically calculated as a function of the LPG period; as a result, the coupled mode was identified as the $\mathrm{LP}_{09}$. The calculations are compared with measured data in Fig. 3. In our experiment, this pair of concatenated LPGs is the auxiliary system that will be used for the spectral characterization of the special FBG recorded in between these LPGs.

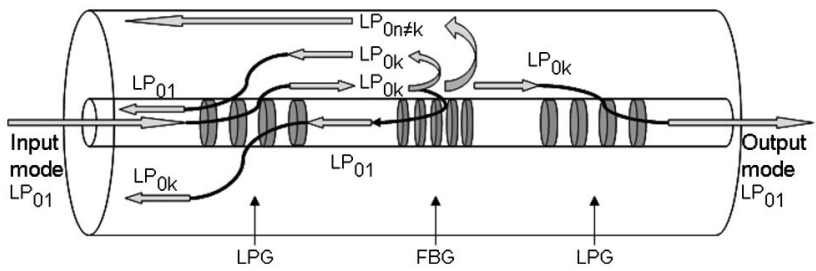

Fig. 1. Schematic diagram of the fiber modes: FBG, fiber Bragg grating; LPG, long period grating. 


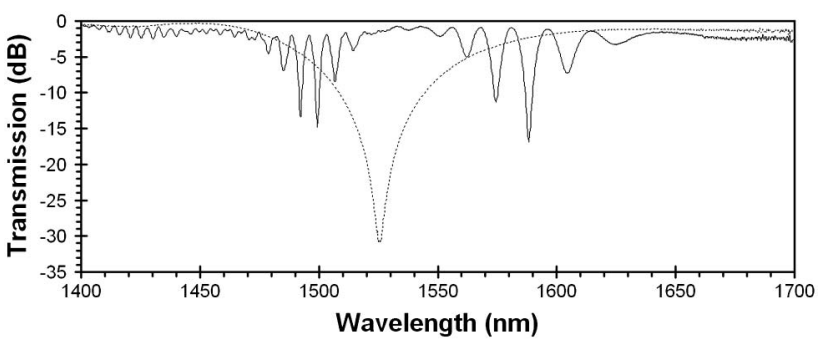

(a)

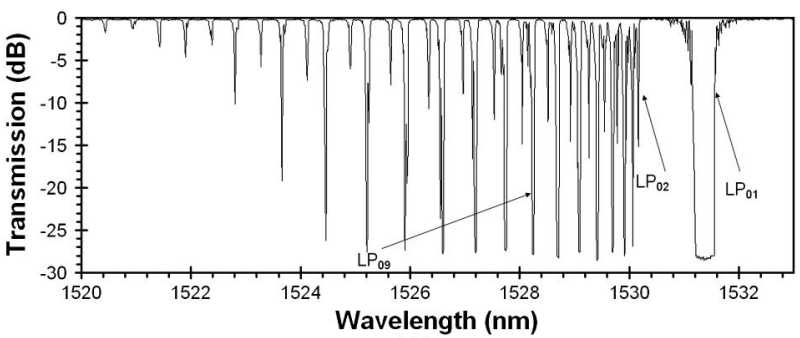

(b)

Fig. 2. (a) Transmission spectra of the LPGs: dotted curve, single grating; solid curve, pair of gratings. (b) Bragg grating transmission spectrum.

A strong Bragg grating $12.1 \mathrm{~cm}$ long was written between the two LPGs scanning the UV beam through a phase mask. The phase mask period was chosen to be $1058 \mathrm{~nm}$, and the fiber was subjected to appropriate strain in order to match the excitation of the high-order cladding modes with the spectral resonance of the LPGs. The Bragg grating spectrum was measured after fabrication by covering the LPGs with an oil layer that avoids energy coupling to cladding modes. The spectrum shown in Fig. 2(b) was measured by a tunable laser scanning in wavelength with $1 \mathrm{pm}$ step; the plot has been normalized to $0 \mathrm{~dB}$. The FBG has the fundamental Bragg resonance at $1531.40 \mathrm{~nm}$ and a bandwidth of $0.39 \mathrm{~nm}$ at $10 \mathrm{~dB}$. Deep transmission notches (with attenuation larger than $20 \mathrm{~dB}$ ) are observed between 1524 and $1530 \mathrm{~nm}$. These resonances correspond to the coupling of light between

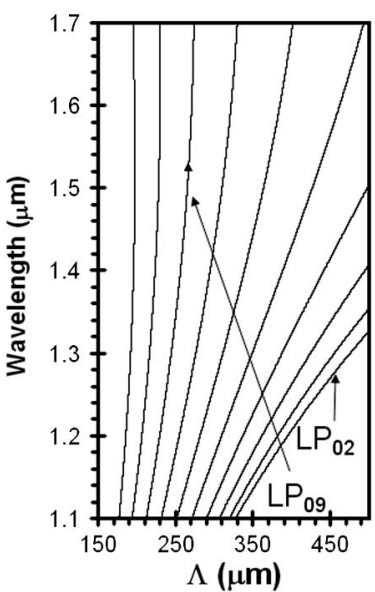

(a)

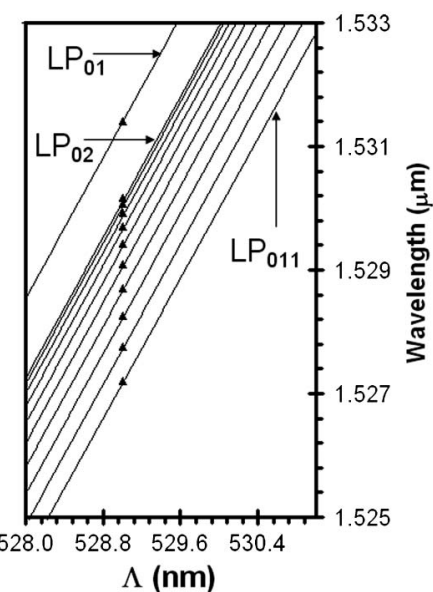

(b)
Fig. 3. (a) Resonant wavelength between the $\mathrm{LP}_{01}$ and $\mathrm{LP}_{0 n}$ copropagating modes versus the LPG period: curves, theory; dot, experiment. (b) Resonant wavelength between the $\mathrm{LP}_{01}$ and the $\mathrm{LP}_{0 n}$ counterpropagating modes versus the Bragg grating period: curves, theory; dots, experiment. the $\mathrm{LP}_{01}$ fundamental core mode and the symmetric cladding modes of odd order, usually labeled " $\mathrm{LP}_{0 n}$ $(n=2,3,4 \ldots)$ cladding modes." Alternated with these resonances is a second set of weaker resonances that corresponds to the cladding modes without axial symmetry that appear despite the small blaze angle owing to the strength of the grating. Symmetric cladding modes of even order are not observed because they have a smaller coupling coefficient than odd modes up to orders of about $n=25[11,15]$. The $\mathrm{LP}_{09}$ mode is coupled by the grating at $1528.25 \mathrm{~nm}$ as is observed in the grating spectrum and theoretically verified in Fig. 3 . This resonance has a bandwidth of $93 \mathrm{pm}$ at $3 \mathrm{~dB}$. The grating strength was estimated in a separate experiment, in which a second grating was written with identical conditions to the first one, and the attenuation of the fundamental and the $\mathrm{LP}_{09}$ resonances were measured as a function of the grating length: the coupling coefficients obtained from the experimental data were $\kappa_{01.01}=5.0 \mathrm{~cm}^{-1}$ and $\kappa_{01,09}=0.70 \mathrm{~cm}^{-1}$. Resonances have a larger bandwidth than predicted for uniform gratings because of the transitions between the grating and the fiber; the two ends of the grating have a lower effective index than the central part, and, therefore, a Fabry-Perot effect broadens the short wavelength edge of the spectrum [17].

When the LPGs are surrounded by air instead of oil, the first grating transfers nearly $100 \%$ of the energy carried by the core mode $\mathrm{LP}_{01}$ to the cladding mode $\mathrm{LP}_{09}$, the Bragg grating couples the cladding mode to

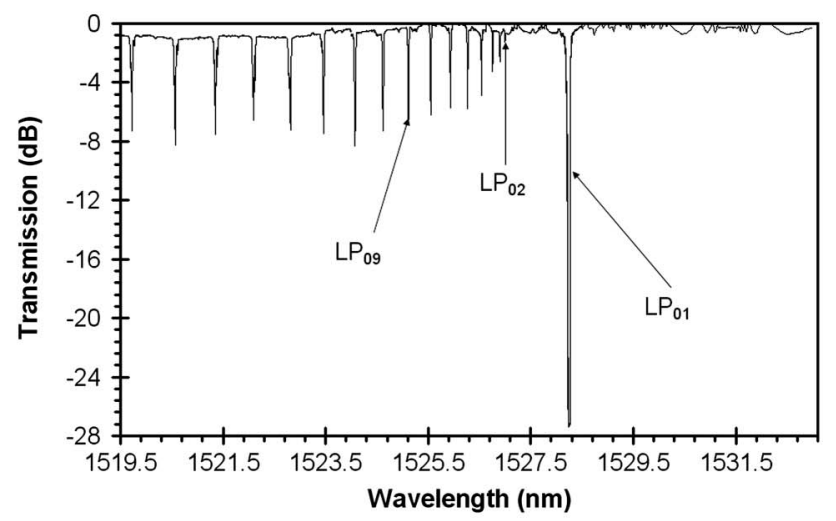

(a)

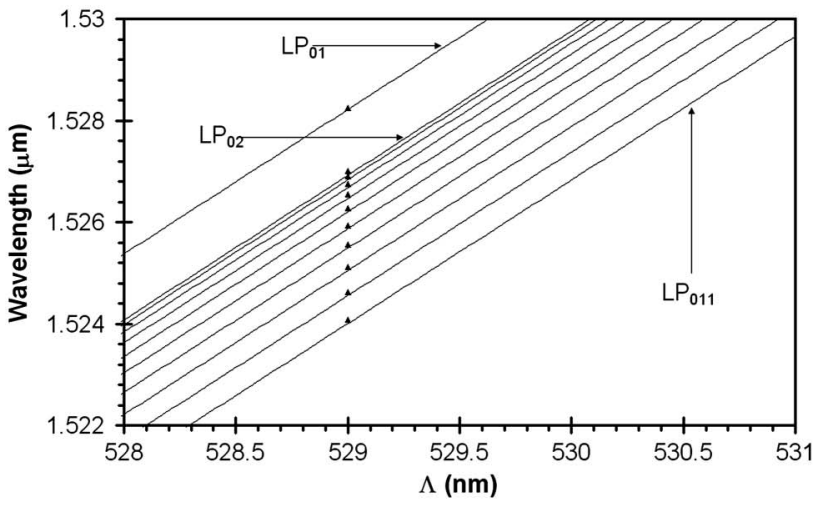

(b)

Fig. 4. (a) Transmission spectrum of the Bragg and LPGs ensemble. (b) Resonances between the $\mathrm{LP}_{09}$ and the $\mathrm{LP}_{0 n}$ counterpropagating modes as a function of the Bragg grating period: curves, theory; dots, experiment. 
Table 1. Coupling Constant $\kappa$ and $3 \mathrm{~dB}$ Bandwidth $\Delta \lambda$ of Resonances between the $\operatorname{LP}_{09}$ and the $\mathrm{LP}_{0 n} \mathrm{Modes}$

\begin{tabular}{lcccccccccccccccc}
\hline$n$ & 1 & 2 & 3 & 4 & 5 & 6 & 7 & 8 & 9 & 10 & 11 & 12 & 13 & 14 & 15 \\
\hline$\kappa\left(\mathrm{cm}^{-1}\right)$ & 0.70 & 0.03 & 0.06 & 0.07 & 0.09 & 0.10 & 0.11 & 0.11 & 0.11 & 0.12 & 0.13 & 0.12 & 0.11 & 0.10 & 0.12 \\
$\Delta \lambda(\mathrm{pm})$ & 93 & - & - & - & 5 & 9 & 17 & 19 & 24 & 24 & 30 & 25 & 34 & 38 & 32 \\
\hline
\end{tabular}

backward traveling modes, and the LPG located downstream reverts the remaining energy of the $\mathrm{LP}_{09}$ mode to the fiber core within a band larger than $29 \mathrm{~nm}$ around the fundamental resonance of the Bragg grating. Thus the transmission spectrum at the fiber output has a set of notches at the wavelengths reflected by the Bragg grating as it can be seen in Fig. 4. The deepest notch at $1528.25 \mathrm{~nm}$ corresponds to the coupling from the $\mathrm{LP}_{09}$ propagating mode to the $\mathrm{LP}_{01}$ counterpropagating mode. This resonance is identical to the ninth resonance of the Bragg grating in Fig. 2. Cladding modes have an effective refractive index smaller than the fundamental core mode; hence, resonances between cladding modes must appear at wavelengths shorter than those associated to the cladding-to-core coupling. Notches at wavelengths below $1528.25 \mathrm{~nm}$ in Fig. 4 correspond to the coupling between the $\mathrm{LP}_{09}$ forward propagating mode and the $\mathrm{LP}_{0 n}$ backward propagating modes (including the $\left.\mathrm{LP}_{09}\right)$. To verify this fact, the coupling wavelengths between cladding modes were computed, and, as can be seen in Fig. 4, they are in good agreement with the experiment. The $3 \mathrm{~dB}$ bandwidth of these resonances is around $25 \mathrm{pm}$, and the coupling coefficients between the $\mathrm{LP}_{09}$ and $\mathrm{LP}_{0 n}(n \geq 2)$ computed from the notch depth is around $0.12 \mathrm{~cm}^{-1}$, as is detailed in Table 1 . As expected, the coupling coefficient between the cladding modes is much smaller than between the cladding and core modes. A small power undulation around $1531.4 \mathrm{~nm}$ can be observed in the spectrum, corresponding to the fundamental resonance of the Bragg grating. The weakness of this spectral perturbation is an indication of the negligible power carried by the forward propagating $\mathrm{LP}_{01}$ mode in the Bragg grating as a consequence of the good performance of the LPG.

Finally, we must point out that the operation principle of the refractometer presented in [10] is not based on the coupling between cladding modes as claimed in that work, but on the coupling between the core and cladding modes. As we have demonstrated here, if the spectral lines of cladding-to-cladding coupling had been observed, a stronger coupling line between the core and the cladding mode would have been observed as well. This idea is also supported by [12], where the device used in [10] is theoretically and experimentally analyzed in terms of the core-to-cladding coupling. Our comment does not restrict the validity of the experimental results reported in [10], which is an excellent experimental paper, we are only trying to make clear which fiber modes are involved in that experiment.
In conclusion, coupling between cladding modes by FBGs has been demonstrated by using a strong grating with a coupling coefficient of $\kappa_{01.01}=5.0 \mathrm{~cm}^{-1}$ and of $12.1 \mathrm{~cm}$ length. Energy transfer between the $\mathrm{LP}_{09}$ and the $\mathrm{LP}_{0 n}(n \geq 1)$ counterpropagating modes has been measured with the assistance of two LPGs. Resonances having $30 \mathrm{pm}$ bandwidth and $8 \mathrm{~dB}$ rejection have been observed, and the coupling coefficient of these resonances has been estimated to be of the order of $0.12 \mathrm{~cm}^{-1}$. Our experiments provide a new tool for the development of an efficient in-fiber mode engineering.

This work was funded by the Ministerio de Ciencia e Innovación (code TEC2008-05490) and the Generalitat Valenciana of Spain (code PROMETEO/2009/077).

\section{References}

1. K. O. Hill, Y. Fujii, D. C. Johnson, and B. S. Kawasaki, Appl. Phys. Lett. 32, 647 (1978).

2. J. N. Blake, B. Y. Kim, and H. J. Shaw, Opt. Lett. 11, 177 (1986).

3. R. Kashyap, Fiber Bragg Gratings (Academic, 1999).

4. K. O. Hill, B. Malo, K. A. Vineberg, F. Bilodeau, D. C. Johnson, and I. Skinner, Electron. Lett. 26, 1270 (1990).

5. S. Ramachandran, B. Mikkelsen, L. C. Cowsar, M. F. Yan, G. Raybon, L. Boivin, M. Fishteyn, W. A. Reed, P. Wisk, D. Brownlow, R. G. Huff, and L. Gruner-Nielsen, IEEE Photon. Technol. Lett. 13, 632 (2001).

6. A. M. Vengsarkar, P. J. Lemaire, J. B. Judkins, V. Bhatia, T. Erdogan, and J. E. Sipe, J. Lightwave Technol. 14, 58 (1996).

7. B. J. Eggleton, P. S. Westbrook, R. S. Windeler, S. Spalter, and T. A. Strasser, Opt. Lett. 24, 1460 (1999).

8. L. Mosquera, D. Sáez-Rodriguez, J. L. Cruz, and M. V. Andrés, Opt. Lett. 35, 613 (2010).

9. L. Y. Shao, A. Laronche, M. Smietana, P. Mikulic, W. J. Bock, and J. Albert, Opt. Commun. 283, 2690 (2010).

10. M. Han, F. Guo, and Y. Lu, Opt. Lett. 35, 399 (2010).

11. T. Erdogan, J. Opt. Soc. Am. A 14, 1760 (1997).

12. A. P. Zhang, X. M. Tao, W. H. Chung, B. O. Guan, and H. Y. Tam, Opt. Lett. 27, 1214 (2002).

13. S. Suzuki, A. Schülzgen, and N. Peyghambarian, Opt. Lett. 33, 351 (2008)

14. R. S. Quimby, T. F. Morse, R. L. Shubochkin, and S. Ramachandran, IEEE J. Sel. Top. Quantum Electron. 15, 12 (2009).

15. X. Shu, K. Sudgen, and I. Bennion, Meas. Sci. Technol. 21, 094003 (2010).

16. A. P. Zhang, H. Y. Tam, and X. M. Tao, Opt. Lett. 28, 519 (2003).

17. V. Mizrahi and J. E. Shipe, J. Lightwave Technol. 11, 1513 (1993). 